\title{
Hamiltonian approach to the ac Josephson effect in superconducting-normal hybrid systems
}

\author{
Qing-feng Sun and Hong Guo \\ Center for the Physics of Materials and Department of Physics, McGill University, Montreal, Québec, Canada H3A 2T8 \\ Jian Wang \\ Department of Physics, University of Hong Kong, Pokfulam Road, Hong Kong, China
}

(Received 9 August 2001; published 30 January 2002)

\begin{abstract}
The ac Josephson effect in hybrid systems of a normal mesoscopic conductor coupled to two superconducting $(S)$ leads is investigated theoretically. A general formula of the ac components of time-dependent current is derived, which is valid for arbitrary interactions in the normal region. We apply this formula to analyze a $S$-normal-S system where the normal region is a noninteracting single-level quantum dot. We report the physical behavior of time-averaged nonequilibrium distribution of electrons in the quantum dot, the formation of Andreev resonance states, and ac components of the time-dependent current. The distribution is found to exhibit a population inversion; and all Andreev resonance states between the superconducting gap $\Delta$ carry the same amount of the current and in the same flow direction. The ac components of time-dependent current show strong oscillatory behavior in marked contrast to the subharmonic gap structure of the average current.
\end{abstract}

DOI: 10.1103/PhysRevB.65.075315

\section{INTRODUCTION}

Quantum-transport properties of mesoscopic conductors coupled to two superconducting $(S)$ leads have been extensively investigated in the last decade both theoretically and experimentally. ${ }^{1,2}$ The mesoscopic conductor in question is usually not a superconductor itself, but it can be a quantum point contact (QPC), ${ }^{3-5}$ a quantum dot (QD), ${ }^{6-9}$ a tunnel barrier, a normal metal, ${ }^{10,11}$ and even a molecule, such as a nanotube. ${ }^{12-14}$ The physics of these hybrid device structures, in the form of $S$-normal- $S$, has profound implications to both fundamental understanding of the quantum transport at a reduced dimensionality and to practical applications in nanoelectronics.

One of the main transport characteristics of a $S$-normal- $S$ device structure is that particles in the normal region can undergo multiple Andreev reflections by the two superconducting leads. If the normal region is ballistic, a consequence of the coherent superposition of these multiple Andreev reflections is the formation of Andreev bound states. ${ }^{1,15}$ The Andreev bound states are important because they carry current including the supercurrent. On the other hand, if the normal region is diffusive, a so-called supercurrent-carrying density of states, instead of the Andreev bound states, gives the ability for carrying supercurrent. ${ }^{16}$ The multiple Andreev reflection is also known to generate subharmonic gap structure in the behavior of $I_{0}=I_{0}(V)$, where $I_{0}$ is the average current and $V$ is the bias voltage. ${ }^{3-8,10}$ More recently, the subharmonic gap structure is used to measure transmission probability of each channel in a multichannel QPC device. $^{17,18}$

Another important and interesting transport characteristic of $S$-normal-S devices is the Josephson effect that gives rise to a dc supercurrent at zero bias, and an ac current at nonzero bias. Previous theoretical analyses have focused on the dc Josephson effect at zero bias, ${ }^{9}$ and the subharmonic gap structure of the average current at a nonzero bias. ${ }^{3-8,10}$ However, the ac Josephson effect, which arises at a nonzero bias, produces a current that is a function of time $t$. There-
PACS number(s): 73.40.Gk, 74.50.+r, 73.23.-b, 72.15.Nj

fore, it is an important task to theoretically understand the time-dependent current in addition to understanding its time average. So far there have been some literatures that report time-dependent current for $S$-normal- $S$ devices., $4,19-22$ For example, Cuevas et al. have investigated the ac component of the time-dependent current for a $S$-QPC- $S$ system; ${ }^{4}$ Bratus et al. ${ }^{5}$ have investigated the time-dependent current in a $S$-quantum-constriction- $S$ system by considering an arbitrary normal electron transparency and discussed the property of the current at the small bias limit; Hurd et al. have studied the ac Josephson current in a device in which the two superconducting leads have different superconducting gaps. $^{21,22}$ Recently, some efforts have also been devoted to the study of time-dependent current in a $d$-wave superconducting system. ${ }^{23,24}$ However, in these previous works, the normal region of the $S$-normal- $S$ device was simplified to a single barrier or a single impurity, and electron-electron interaction in the normal region has been neglected. The superconducting lead may be closely coupled to the barrier ${ }^{4,5}$ or with a certain distance. ${ }^{21,22}$ The barrier has been described by a simple constant transmission coefficient independent of energy $\epsilon$. Given the interesting physics already discovered by these previous investigations, it is indeed not difficult to expect that even richer physics would arise if the normal region has its own electronic structure and/or electron-electron interactions.

It is the purpose of this work to further investigate the ac Josephson effect in $S$-normal- $S$ device systems, and we focus on issues not resolved by the previous analysis. In particular, we consider a mesoscopic $S$-normal- $S$ device with an arbitrary normal region that may have its own electronic structure and/or strong electron-electron interactions: for this general situation we have derived the expression of the ac current. As an application we then investigate a specific case in which the normal region is a ballistic quantum dot (i.e., a double-barriers structure) having a noninteracting single energy level, for which we investigate the intradot nonequilibrium distribution of electrons, the local density of state 
(LDOS), and the time-dependent current. Our main findings are as follows.

(i) The intradot electronic distribution shows a population inversion property. This property is distinctly and qualitatively different from that of the case where the normal region is diffusive.

(ii) At small bias voltages such that $e V<\Delta$, where $\Delta$ is the superconducting gap, a series of Andreev resonance states is found to emerge within the gap. Their weights are not the same but they carry equal amount of current in the same direction, as well, their electronic distributions are all $1 / 2$.

(iii) The ac current component versus bias $V$ shows an oscillatory behavior. The amplitude of oscillation of the $n$th component is largest at about $V=\Delta / n$. At small bias, the high-order components quickly increase, and the timedependent current versus time $t$ deviates from a sinelike curve.

The rest of the paper is organized as follows. In Sec. II, the model Hamiltonian is presented and a general formula for the ac current component is derived. In Sec. III, ac Josephson effect for a simple $S$-normal-S device with a noninteracting normal region is investigated. The intradot electronic distributions, the Andreev resonance states, and ac current components are presented in this section. Finally, a brief summary is given in Sec. IV.

\section{MODEL AND FORMULATION}

We assume the $S$-normal- $S$ device system to be described by the following Hamiltonian: ${ }^{25,26}$

$$
H=\sum_{\alpha=L, R} H_{\alpha}+H_{c e n}+H_{T}
$$

where

$$
\begin{gathered}
H_{\alpha}=\sum_{k, \sigma} \epsilon_{\alpha k} a_{\alpha k \sigma}^{\dagger} a_{\alpha k \sigma} \\
+\sum_{k}\left[\Delta_{\alpha} a_{\alpha k \downarrow} a_{\alpha-k \uparrow}+\Delta_{\alpha} a_{\alpha-k \uparrow}^{\dagger} a_{\alpha k \downarrow}^{\dagger}\right], \\
H_{c e n}=\sum_{j, \sigma} \epsilon_{j \sigma} c_{j \sigma}^{\dagger} c_{j \sigma}+H_{i n t}\left(\left\{c_{j \sigma}^{\dagger}\right\},\left\{c_{j \sigma}\right\}, \ldots\right), \\
H_{T}=\sum_{k, j, \sigma, \alpha} t_{\alpha j} \exp \left[\frac{i}{2}\left(\phi_{\alpha}+\frac{2 e V_{\alpha}}{\hbar} t\right)\right] a_{\alpha k \sigma}^{\dagger} c_{j \sigma}+\text { H.c. }
\end{gathered}
$$

$H_{\alpha}(\alpha=L, R)$ describes the left/right BCS superconducting lead with the superconducting energy gap $\Delta_{\alpha} . H_{c e n}$ is the Hamiltonian of the normal region of the device, and $c_{j \sigma}^{\dagger}\left(c_{j \sigma}\right)$ are the creation (annihilation) operators of an electron in state $j \sigma$ of the normal region. $H_{\text {int }}$ models interactions in the normal region whose form depends on specific physics problems under consideration. In this section we consider the general case without specifying its concrete form. In deriving the formula for the transport current, we permit the device-normal (central) region to have various interactions, such as the electron-electron Coulomb interaction, $\sum_{j, \sigma ; j_{1}, \sigma_{1}\left(j \sigma \neq j_{1} \sigma_{1}\right)} U_{j \sigma ; j_{1} \sigma_{1}} c_{j \sigma}^{\dagger} c_{j \sigma} c_{j_{1} \sigma_{1}}^{\dagger} c_{j_{1} \sigma_{1}}$; the electron-phonon interaction, $\quad \sum_{j, \sigma, q} M_{j q} c_{j \sigma}^{\dagger} c_{j \sigma}\left(d_{q}^{\dagger}+d_{-q}\right)$ $+\Sigma_{q} \hbar \omega_{q} d_{q}^{\dagger} d_{q}$; the tunneling coupling between different states of the normal region, $\Sigma_{i, j, \sigma(i>j)}\left(t_{i j} c_{i \sigma}^{\dagger} c_{j \sigma}+\right.$ H.c. $)$; and so on. $H_{T}$ of Eq. (1) denotes the tunneling Hamiltonian between the superconducting leads and the normal region of the device, and $t_{\alpha j}$ is the hopping matrix. In order to obtain the Hamiltonian (1), we have performed a unitary transformation, then the superconducting initial phase $\phi_{\alpha}$ and the terminal voltage $V_{\alpha}$ emerge in the Hamiltonian $H_{T} \cdot{ }^{25,26}$

The total current of superconducting lead $\alpha$ (e.g., $\alpha=L$ ) flowing into the device-normal region can be calculated from evolution of the total number operator of electrons in that lead, $N_{L}=\sum_{k, \sigma} a_{L k \sigma}^{\dagger} a_{L k \sigma}$. Then we have $e^{26-28}$ (in units of $\hbar=1)$

$$
\begin{aligned}
I_{L}(t) & =-e\left\langle\dot{N}_{L}(t)\right\rangle=i e\left\langle\left[N_{L}, H\right]\right\rangle \\
& =2 e \operatorname{Re} \sum_{k, i} \operatorname{Tr}\left\{\hat{G}_{i, L k}^{<}(t, t) \hat{t}_{L i}(t) \hat{\sigma}_{z}\right\},
\end{aligned}
$$

where

$$
\hat{G}_{i, L k}^{<}\left(t, t_{1}\right) \equiv i\left(\begin{array}{cc}
\left\langle a_{L k \uparrow}^{\dagger}\left(t_{1}\right) c_{i \uparrow}(t)\right\rangle & \left\langle a_{L-k \downarrow}\left(t_{1}\right) c_{i \uparrow}(t)\right\rangle \\
\left\langle a_{L k \uparrow}^{\dagger}\left(t_{1}\right) c_{i \downarrow}^{\dagger}(t)\right\rangle & \left\langle a_{L-k \downarrow}\left(t_{1}\right) c_{i \downarrow}^{\dagger}(t)\right\rangle
\end{array}\right)
$$

is the distribution Green's function in the $2 \times 2$ Nambu representation, and $\hat{\sigma}_{z}$ is the Pauli matrix. In this paper, we use the notation that " $\hat{A}$ " means quantity $A$ to be a $2 \times 2$ matrix.

To proceed we need to solve the Green's function $\hat{G}_{i, L k}^{<}(t, t)$. We assume that the leads do not have any interactions except the quadratic pair potential correlation, we have $^{26,28}$

$$
\begin{aligned}
\hat{G}_{i, L k}^{<}(t, t)= & \sum_{j} \int d t_{1}\left[\hat{G}_{i j}^{r}\left(t, t_{1}\right) \hat{t}_{L j}^{*}\left(t_{1}\right) \hat{g}_{L k}^{<}\left(t_{1}, t\right)\right. \\
& \left.+\hat{G}_{i j}^{<}\left(t, t_{1}\right) \hat{t}_{L j}^{*}\left(t_{1}\right) \hat{g}_{L k}^{a}\left(t_{1}, t\right)\right]
\end{aligned}
$$

where $\hat{g}_{L k}^{<, a}\left(t_{1}, t\right)$ is the exact Green's function of the left superconducting lead. ${ }^{4,25} \hat{t}_{L j}(t)$ in Eqs. (5) and (6) is a $2 \times 2$ hopping matrix defined by

$$
\hat{t}_{L j}(t)=\left(\begin{array}{ll}
t_{L j} \exp \left[i\left(\frac{\phi_{L}}{2}+e V_{L} t\right)\right] & 0 \\
0 & -t_{L j}^{*} \exp \left[-i\left(\frac{\phi_{L}}{2}+e V_{L} t\right)\right]
\end{array}\right) .
$$


$\hat{G}_{i j}^{r}\left(t, t_{1}\right)$ and $\hat{G}_{i j}^{<}\left(t, t_{1}\right)$ are the retarded and distribution Green's functions in the device normal region. They are defined by

$$
\begin{aligned}
& \hat{G}_{i j}^{r}\left(t, t_{1}\right)=-i \theta\left(t-t_{1}\right) \\
& \times\left(\begin{array}{cc}
\left\langle\left\{c_{i \uparrow}(t), c_{j \uparrow}^{\dagger}\left(t_{1}\right)\right\}\right\rangle & \left\langle\left\{c_{i \uparrow}(t), c_{j \downarrow}\left(t_{1}\right)\right\}\right\rangle \\
\left\langle\left\{c_{i \downarrow}^{\dagger}(t), c_{j \uparrow}^{\dagger}\left(t_{1}\right)\right\}\right\rangle & \left\langle\left\{c_{i \downarrow}^{\dagger}(t), c_{j \downarrow}\left(t_{1}\right)\right\}\right\rangle
\end{array}\right), \\
& \hat{G}_{i j}^{<}\left(t, t_{1}\right)=i\left(\begin{array}{ll}
\left\langle c_{j \uparrow}^{\dagger}\left(t_{1}\right) c_{i \uparrow}(t)\right\rangle & \left\langle c_{j \downarrow}\left(t_{1}\right) c_{i \uparrow}(t)\right\rangle \\
\left\langle c_{j \uparrow}^{\dagger}\left(t_{1}\right) c_{i \downarrow}^{\dagger}(t)\right\rangle & \left\langle c_{j \downarrow}\left(t_{1}\right) c_{i \downarrow}^{\dagger}(t)\right\rangle
\end{array}\right)
\end{aligned}
$$

Substituting $\hat{G}_{j, L k}^{<}(t, t)$ into Eq. (5), assuming $t_{L j}$ is real, the current $I_{L}(t)$ can be expressed in terms of the Green's functions of the device normal region, as

$$
\hat{\Sigma}_{L}(\epsilon)=\left(\begin{array}{ll}
\exp \left[-i e V_{L}\left(t_{1}-t\right)\right] & -\frac{\Delta}{\epsilon} \exp \left[-i \phi_{L}-i e V_{L}\left(t_{1}+t\right)\right] \\
-\frac{\Delta}{\epsilon} \exp \left[i \phi_{L}+i e V_{L}\left(t_{1}+t\right)\right] & \exp \left[i e V_{L}\left(t_{1}-t\right)\right]
\end{array}\right)
$$

$$
\begin{aligned}
I_{L}(t)= & -2 e \operatorname{Im} \int_{-\infty}^{t} d t_{1} \int \frac{d \epsilon}{2 \pi} e^{i \epsilon\left(t-t_{1}\right)} \operatorname{Tr}\left\{\left[\widetilde{\rho}_{L}(\epsilon) f_{L}(\boldsymbol{\epsilon})\right.\right. \\
& \left.\left.\times \hat{\mathbf{G}}^{r}\left(t, t_{1}\right)+\beta_{L}^{*}(\boldsymbol{\epsilon}) \hat{\mathbf{G}}^{<}\left(t, t_{1}\right)\right] \boldsymbol{\Gamma}_{L} \hat{\Sigma}_{L} \hat{\sigma}_{z}\right\}
\end{aligned}
$$

where $f_{L / R}(\epsilon)=1 /\left(e^{\epsilon / K_{B}} \mathcal{T}+1\right)$ is the Fermi distribution function of electrons in the left/right superconducting lead. $\beta_{L}(\epsilon)$ is defined as ${ }^{25,29} \beta_{L}(\epsilon)=\epsilon /\left(i \sqrt{\Delta_{L}^{2}-\epsilon^{2}}\right)$ for $\Delta_{L}>|\epsilon|$, and $\beta_{L}(\epsilon)=|\epsilon| /\left(\sqrt{\epsilon^{2}-\Delta_{L}^{2}}\right) \quad$ for $\quad \Delta_{L}<|\epsilon| . \quad \widetilde{\rho}_{L}(\epsilon)=\operatorname{Re}\left[\beta_{L}(\epsilon)\right]$ $=\theta(|\epsilon|-\Delta)|\epsilon| / \sqrt{\left(\epsilon^{2}-\Delta_{L}^{2}\right)}$ is the dimensionless BCS density of states, i.e., the ratio of the superconducting density of states $\rho_{L}^{S}(\epsilon)$ to the normal density of states $\rho_{L}^{N}(\epsilon)$. $\Gamma$ is the linewidth matrix function defined by $\Gamma_{L ; i j}=2 \pi t_{L i} t_{L j}^{*} \rho_{L}^{N}(\epsilon)$, in which we have assumed that $\boldsymbol{\Gamma}_{L}$ is independent energy $\epsilon^{30}$ In this paper, we use boldface letters to denote quantities representing matrices whose matrix elements are calculated using states $i, j$ of the device-normal region. Finally, $\hat{\Sigma}_{L}$ is a compact notation,
The formula (10) describes the current using Green's functions of the normal region. It is a general formula and can, therefore, be applied to situations involving arbitrary interactions in the normal region and is also applicable at nonequilibrium (e.g., at a high bias $V$ ). If the normal region is coupled to multiple superconducting leads or to some extra normal leads, Eq. (10) is still valid.

In the following we fix $V_{L}=0,{ }^{31}$ so that the left superconducting lead is taken as the potential ground, then $\hat{\Sigma}_{L}$ reduces to

$$
\hat{\Sigma}_{L}(\epsilon)=\left(\begin{array}{ll}
1 & -\frac{\Delta}{\epsilon} e^{-i \phi_{L}} \\
-\frac{\Delta}{\epsilon} e^{i \phi_{L}} & 1
\end{array}\right)
$$

Note that the superconducting phase difference between the two leads is a time-dependent periodic function with a period $T=2 \pi / \omega$, where $\omega=2 \mathrm{eV}$ and $V=V_{L}-V_{R}$ is the bias voltage between the leads. Therefore, the time-dependent current $I_{L}(t)$ is also a periodic function with the same period $T$ and the Green's functions have the property $G\left(t, t_{1}\right)=G(t$ $\left.+T, t_{1}+T\right) .^{32}$ Then we can take the conventional Fourier expansion for the current $I_{L}(t)$

$$
I_{L}(t)=\sum_{n} I_{L n} e^{i n \omega t},
$$

and take the double Fourier expansion for the Green's function $^{4,30}$

$$
G\left(t, t_{1}\right)=\sum_{n} e^{i n \omega t_{1}} \int \frac{d \epsilon}{2 \pi} e^{-i \epsilon\left(t-t_{1}\right)} G_{n}(\epsilon) .
$$

To simplify notation in the following analysis, we introduce quantities $G_{m n}(\epsilon) \equiv G_{n-m}(\epsilon+m \omega)$ and $\mathcal{I}_{L}(t)$,

$$
\begin{aligned}
\mathcal{I}_{L}(t)= & -2 e \int_{-\infty}^{t} d t_{1} \int \frac{d \epsilon}{2 \pi} e^{i \epsilon\left(t-t_{1}\right)} \operatorname{Tr}\left\{\left[\widetilde{\rho}_{L}(\epsilon) f_{L}(\epsilon) \hat{\mathbf{G}}^{r}\left(t, t_{1}\right)\right.\right. \\
& \left.\left.+\beta_{L}^{*}(\epsilon) \hat{\mathbf{G}}^{<}\left(t, t_{1}\right)\right] \boldsymbol{\Gamma}_{L} \hat{\Sigma}_{L} \hat{\sigma}_{z}\right\},
\end{aligned}
$$

so that $I_{L}(t)=\operatorname{Im}\left[\mathcal{I}_{L}(t)\right]$.

Then the Fourier component of ac current is obtained as

$$
I_{L n}=\frac{i}{2}\left(\mathcal{I}_{L,-n}^{*}-\mathcal{I}_{L n}\right)
$$

and

$$
\begin{aligned}
\mathcal{I}_{L n}= & -2 e \int \frac{d \epsilon}{2 \pi} \operatorname{Tr}\left\{\left[f_{L}(\epsilon) \widetilde{\rho}_{L}(\epsilon) \hat{\mathbf{G}}_{-n 0}^{r}(\epsilon)\right.\right. \\
& \left.\left.+\frac{1}{2} \beta_{L}^{*}(\epsilon) \hat{\mathbf{G}}_{-n 0}^{<}(\epsilon)\right] \boldsymbol{\Gamma}_{L} \hat{\Sigma}_{L} \hat{\sigma}_{z}\right\} .
\end{aligned}
$$

Equations (16) and (17) are the first central results of this work. They describe ac components of the time-dependent 
current of a $S$-normal- $S$ device system in terms of the Fourier component of the Green's function $\hat{\mathbf{G}}_{-n 0}^{r}(\boldsymbol{\epsilon})$ and $\hat{\mathbf{G}}_{-n 0}^{<}(\boldsymbol{\epsilon})$ of the normal region. These formulas, Eqs. (13), (16), and (17), are valid for arbitrary interactions the normal region may have, for nonequilibrium situations, and for devices with other normal leads. They cannot, however, be applied to devices with more than two superconducting leads.

When bias voltage $V$ is zero the current $I_{L}(t)$ is independent to time $t$, then the current reduces as

$$
\begin{aligned}
I_{L}= & -2 e \operatorname{Im} \int \frac{d \boldsymbol{\epsilon}}{2 \pi} \operatorname{Tr}\left\{\left[f_{L}(\boldsymbol{\epsilon}) \tilde{\rho}_{L}(\boldsymbol{\epsilon}) \hat{\mathbf{G}}^{r}(\boldsymbol{\epsilon})\right.\right. \\
& \left.\left.+\frac{1}{2} \beta_{L}^{*}(\boldsymbol{\epsilon}) \hat{\mathbf{G}}^{<}(\boldsymbol{\epsilon})\right] \boldsymbol{\Gamma}_{L} \hat{\tilde{\Sigma}}_{L} \hat{\sigma}_{z}\right\} .
\end{aligned}
$$

\section{NONINTERACTING NORMAL REGION}

In this section we apply the general expressions for the ac current derived above to an example of a $S$-normal- $S$ device where the normal region has no electron-electron interactions. For this situation, the Hamiltonian $H_{c e n}$ can be written as

$$
\begin{aligned}
H_{c e n} & =\sum_{j, \sigma} \epsilon_{j \sigma} c_{j \sigma}^{\dagger} c_{j \sigma}+\sum_{i, j, \sigma(i>j)}\left(t_{i j} c_{i \sigma}^{\dagger} c_{j \sigma}+\text { H.c. }\right) \\
& \equiv \sum_{\sigma} H_{c e n, \sigma} .
\end{aligned}
$$

This Hamiltonian describes a multilevel noninteracting quantum dot for which $t_{i j}=0$. It also can describe a typical tightbinding lattice model, in which $t_{i j} \neq 0$, the second term in Eq. (19) denotes the coupling between different lattice sites.

For the specific $H_{c e n}$ of Eq. (19), we can solve the
Green's functions $\hat{\mathbf{G}}_{m n}^{r}(\boldsymbol{\epsilon})$ and $\hat{\mathbf{G}}_{m n}^{<}(\boldsymbol{\epsilon})$ using the Dyson equation and the Keldysh equation: $\hat{\mathbf{G}}^{r}=\hat{\mathbf{g}}^{r}+\hat{\mathbf{G}}^{r} \hat{\boldsymbol{\Sigma}}^{r} \hat{\mathbf{g}}^{r}$, and $\hat{\mathbf{G}}^{<}=\hat{\mathbf{G}}^{r} \hat{\mathbf{\Sigma}}^{<} \hat{\mathbf{G}}^{a}$. Here $\hat{\mathbf{g}}^{r}$ is the exact Green's function for the device-normal region without coupling to the leads, and it can be easily derived as

$$
\begin{aligned}
\hat{\mathbf{g}}^{r}\left(t, t_{1}\right)= & -i \theta\left(t-t_{1}\right) \\
& \times\left(\begin{array}{ll}
\exp \left[-i H_{c e n \uparrow}\left(t-t_{1}\right)\right] & 0 \\
0 & \exp \left[i H_{c e n \downarrow}\left(t-t_{1}\right)\right]
\end{array}\right) .
\end{aligned}
$$

$\hat{\mathbf{\Sigma}}^{r}$ and $\hat{\mathbf{\Sigma}}^{<}$are the retarded and distribution self-energies due to coupling to the leads, with $\hat{\boldsymbol{\Sigma}}^{r(<)}\left(t, t_{1}\right)=\hat{\boldsymbol{\Sigma}}_{L}^{r(<)}\left(t, t_{1}\right)$ $+\hat{\mathbf{\Sigma}}_{R}^{r(<)}\left(t, t_{1}\right)$ and

$$
\begin{aligned}
\hat{\Sigma}_{L(R), i j}^{r}\left(t, t_{1}\right)= & \sum_{k} \hat{t}_{L(R) i}^{*}(t) \hat{g}_{L(R) k}^{r}\left(t, t_{1}\right) \hat{t}_{L(R) j}\left(t_{1}\right) \\
= & -i \theta\left(t-t_{1}\right) \int \frac{d \epsilon}{2 \pi} \Gamma_{L(R), i j} \beta_{L(R)}(\epsilon) \\
& \times e^{-i \epsilon\left(t-t_{1}\right)} \hat{\tilde{\Sigma}}_{L(R)}, \\
\hat{\Sigma}_{L(R), i j}^{<}\left(t, t_{1}\right)= & \sum_{k} \hat{t}_{L(R) i}^{*}(t) \hat{g}_{L(R) k}^{<}\left(t, t_{1}\right) \hat{t}_{L(R) j}\left(t_{1}\right) \\
= & i \int \frac{d \epsilon}{2 \pi} \Gamma_{L(R), i j} f_{L(R)}(\epsilon) \tilde{\rho}_{L(R)}(\epsilon) \\
& \times e^{-i \epsilon\left(t-t_{1}\right)} \hat{\Sigma}_{L(R)} .
\end{aligned}
$$

The Fourier spaces form of these quantities are easily obtained (notice that $V_{L}=0$ and $V_{R}=-V$ )

$$
\begin{aligned}
& \hat{\mathbf{g}}_{m n}^{r}(\boldsymbol{\epsilon})=\left(\begin{array}{ll}
\delta_{m n} /\left(\boldsymbol{\epsilon}_{m}-\mathbf{H}_{c e n \uparrow}+i 0^{+}\right) & 0 \\
0 & \delta_{m n} /\left(\boldsymbol{\epsilon}_{m}+\mathbf{H}_{c e n \uparrow}+i 0^{+}\right)
\end{array}\right), \\
& \hat{\boldsymbol{\Sigma}}_{L ; m n}^{r}(\boldsymbol{\epsilon})=-\frac{i}{2} \boldsymbol{\Gamma}_{L} \delta_{m n} \beta_{L}\left(\boldsymbol{\epsilon}_{m}\right) \hat{\Sigma}_{L}\left(\boldsymbol{\epsilon}_{m}\right), \\
& \hat{\boldsymbol{\Sigma}}_{R ; m n}^{r}(\boldsymbol{\epsilon})=-\frac{i}{2} \boldsymbol{\Gamma}_{R}\left(\begin{array}{ll}
\delta_{m n} \beta_{R}\left(\boldsymbol{\epsilon}_{m+(1 / 2)}\right) & \delta_{m, n-1} \beta_{R}\left(\boldsymbol{\epsilon}_{m+(1 / 2)}\right) \frac{-\Delta_{R}}{\epsilon_{m+(1 / 2)}} e^{-i \phi_{R}} \\
\delta_{m, n+1} \beta_{R}\left(\boldsymbol{\epsilon}_{m-(1 / 2)}\right) \frac{-\Delta_{R}}{\epsilon_{m-(1 / 2)}} e^{i \phi_{R}} & \delta_{m n} \beta_{R}\left(\boldsymbol{\epsilon}_{m-(1 / 2)}\right)
\end{array}\right), \\
& \hat{\boldsymbol{\Sigma}}_{L ; m n}^{<}(\boldsymbol{\epsilon})=i \boldsymbol{\Gamma}_{L} \delta_{m n} f_{L}\left(\boldsymbol{\epsilon}_{m}\right) \widetilde{\rho}_{L}\left(\boldsymbol{\epsilon}_{m}\right) \hat{\Sigma}_{L}\left(\epsilon_{m}\right) \\
& \hat{\boldsymbol{\Sigma}}_{R ; m n}^{<}(\boldsymbol{\epsilon})=i \boldsymbol{\Gamma}_{R}\left(\begin{array}{ll}
\delta_{m n} f_{L}\left(\boldsymbol{\epsilon}_{m+(1 / 2)}\right) \widetilde{\rho}_{R}\left(\boldsymbol{\epsilon}_{m+(1 / 2)}\right) & \delta_{m, n-1} f_{L}\left(\boldsymbol{\epsilon}_{m+(1 / 2)}\right) \widetilde{\rho}_{R}\left(\boldsymbol{\epsilon}_{m+(1 / 2)}\right) \frac{-\Delta_{R}}{\epsilon_{m+(1 / 2)}} e^{-i \phi_{R}} \\
\delta_{m, n+1} f_{L}\left(\boldsymbol{\epsilon}_{m-(1 / 2)}\right) \widetilde{\rho}_{R}\left(\boldsymbol{\epsilon}_{m-(1 / 2)}\right) \frac{-\Delta_{R}}{\boldsymbol{\epsilon}_{m-(1 / 2)}} e^{i \phi_{R}} & \delta_{m n} f_{R}\left(\boldsymbol{\epsilon}_{m-(1 / 2)}\right) \widetilde{\rho}_{R}\left(\boldsymbol{\epsilon}_{m-(1 / 2)}\right)
\end{array}\right),
\end{aligned}
$$


where $\epsilon_{x}=\epsilon+x \omega$. Similarly, the Fourier spaces form of the Keldysh equation and the Dyson equation are

$$
\begin{gathered}
\hat{\mathbf{G}}_{m n}^{<}(\boldsymbol{\epsilon})=\sum_{l_{1}, l_{2}} \hat{\mathbf{G}}_{m l_{1}}^{r}(\boldsymbol{\epsilon}) \hat{\mathbf{\Sigma}}_{l_{1} l_{2}}^{<}(\boldsymbol{\epsilon}) \hat{\mathbf{G}}_{l_{2} n}^{a}(\boldsymbol{\epsilon}), \\
\hat{\mathbf{G}}_{m n}^{r}(\boldsymbol{\epsilon})=\hat{\mathbf{g}}_{m n}^{r}(\boldsymbol{\epsilon}) \delta_{m n}+\sum_{l} \hat{\mathbf{G}}_{m l}^{r}(\boldsymbol{\epsilon}) \hat{\mathbf{\Sigma}}_{l n}^{r}(\boldsymbol{\epsilon}) \hat{\mathbf{g}}_{n n}^{r}(\boldsymbol{\epsilon}) .
\end{gathered}
$$

If $\hat{\mathbf{G}}_{m n}^{r}(\boldsymbol{\epsilon})$ has been solved, then from the Keldysh equation (28), $\hat{\mathbf{G}}_{m n}^{<}(\epsilon)$ can be obtained straightforwardly. Therefore, in the following we only need to solve the retarded Green's function $\hat{\mathbf{G}}_{m n}^{r}(\boldsymbol{\epsilon})$.

From the Dyson equation (29) we have

$$
\begin{gathered}
\mathbf{G}_{m n ; 11}^{r}=\mathbf{g}_{m n ; 11}^{r} \delta_{m n}+\mathbf{G}_{m n ; 11}^{r} \Sigma_{n n ; 11}^{r} \mathbf{g}_{n n ; 11}^{r} \\
+\sum_{l} \mathbf{G}_{m l ; 12}^{r} \boldsymbol{\Sigma}_{l n ; 21}^{r} \mathbf{g}_{n n ; 11}^{r}, \\
\mathbf{G}_{m n ; 12}^{r}=\mathbf{G}_{m n ; 12}^{r} \Sigma_{n n ; 22}^{r} \mathbf{g}_{n n ; 22}^{r}+\sum_{l} \mathbf{G}_{m l ; 11}^{r} \Sigma_{l n ; 12}^{r} \mathbf{g}_{n n ; 22}^{r},
\end{gathered}
$$

where we have suppressed the argument $\epsilon$. From Eq. (31), one has

$$
\mathbf{G}_{m n ; 12}^{r}=\sum_{l} \mathbf{G}_{m l ; 11}^{r} \mathbf{\Sigma}_{l n ; 12}^{r} \frac{1}{\mathbf{g}_{n n ; 22}^{r-1}-\mathbf{\Sigma}_{n n ; 22}^{r}} .
$$

In here, $\mathbf{g}^{r-1}$ is $\left(\mathbf{g}^{r}\right)^{-1}$. Substituting this expression to Eq. (30) one easily finds

$$
\mathbf{G}_{m n ; 11}^{r}=\frac{\delta_{m n}}{\mathbf{g}_{n n ; 22}^{r-1}-\mathbf{\Sigma}_{n n ; 22}^{r}}+\sum_{l} \mathbf{G}_{m l ; 11}^{r} \mathbf{B}_{l n},
$$

where

$$
\mathbf{B}_{m n}(\boldsymbol{\epsilon}) \equiv \sum_{l} \boldsymbol{\Sigma}_{m l ; 12}^{r} \frac{1}{\mathbf{g}_{l l ; 22}^{r-1}-\boldsymbol{\Sigma}_{l l ; 22}^{r}} \boldsymbol{\Sigma}_{l n ; 21}^{r} \frac{1}{\mathbf{g}_{n n ; 11}^{r-1}-\boldsymbol{\Sigma}_{n n ; 11}^{r}}
$$

Note $\mathbf{B}_{m n} \neq 0$ only when $m=n, n \pm 1$. The quantity $\mathbf{B}_{m n}$ has a clear physical meaning: it describes the intensity of Andreev reflection processes, an example is shown in Fig. 1 in which a particle in the normal region undergoes twice Andreev reflections. Then by iterating Eq. (33), $\mathbf{G}_{m n ; 11}^{r}$ can be formally solved,

$$
\mathbf{G}_{m n ; 11}^{r}=\frac{\delta_{m n}}{\mathbf{g}_{n n ; 11}^{r-1}-\mathbf{\Sigma}_{n n ; 11}^{r}}+\frac{1}{\mathbf{g}_{m m ; 11}^{r-1}-\mathbf{\Sigma}_{m m ; 11}^{r}} \mathbf{Y}_{m n},
$$

where

$$
\begin{aligned}
\mathbf{Y}_{m n} & =\mathbf{B}_{m n}+\sum_{l_{1}} \mathbf{B}_{m l_{1}} \mathbf{B}_{l_{1} n}+\sum_{l_{1}, l_{2}} \mathbf{B}_{m l_{1}} \mathbf{B}_{l_{1} l_{2}} \mathbf{B}_{l_{2} n}+\ldots \\
& =\mathbf{B}_{m n}+\sum_{l} \mathbf{B}_{m l} \mathbf{Y}_{l n} .
\end{aligned}
$$

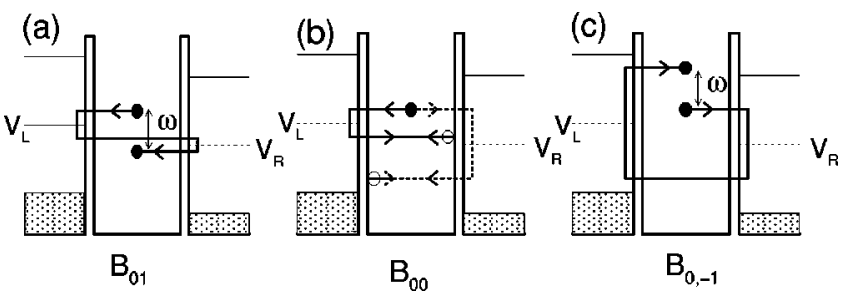

FIG. 1. A schematic diagram for the transport process consisting of two Andreev reflections. (a). The particle is first Andreev reflected by the left superconducting lead, then by the right superconducting lead. This is described by the quantity $\mathbf{B}_{01}(\epsilon)$. After this process, the particle energy reduces by $2 \mathrm{eV}$ (i.e., $\omega=2 \mathrm{eV}$ ). (b). The particle is first Andreev reflected by the left (right) lead, followed by another reflection at the same lead. This process is described by quantity $\mathbf{B}_{00}(\epsilon)$. After this process, the particle energy does not change. (c). The particle is first Andreev reflected by the right lead, then by the left lead. It is described by quantity $\mathbf{B}_{0,-1}(\epsilon)$. After this process, the particle energy rises $2 \mathrm{eV}$. All processes with an even number Andreev reflections can be decomposed to the three processes plotted here. All processes with an odd number of Andreev reflections can be decomposed to the even case plus one more reflection.

Similarly, the quantity $\mathbf{Y}_{m n}(\boldsymbol{\epsilon})$ has a clear physical meaning: it gives the intensity of the process for which an electron having initial energy $\epsilon+n \omega$ ends up with final energy $\epsilon$ $+m \omega$ after going through all possible multiple Andreev reflections in the normal region. Equation (36) can only be solved numerically and after $\mathbf{Y}_{m n}$ is solved, from Eqs. (35) and (32) $\mathbf{G}_{m n ; 11}^{r}$ and $\mathbf{G}_{m n ; 12}^{r}$ can be obtained immediately. Finally, $\mathbf{G}_{m n ; 21}^{r}$ and $\mathbf{G}_{m n ; 22}^{r}$ can also be calculated using the following equations, which are derived from the Dyson equation:

$$
\mathbf{G}_{m n ; 21}^{r}=\sum_{l} \frac{1}{\mathbf{g}_{m m ; 22}^{r-1}-\mathbf{\Sigma}_{m m ; 22}^{r}} \mathbf{\Sigma}_{m l ; 21}^{r} \mathbf{G}_{l n ; 11}^{r}
$$

$$
\mathbf{G}_{m n ; 22}^{r}=\frac{\delta_{m n}}{\mathbf{g}_{m m ; 22}^{r-1}-\mathbf{\Sigma}_{m m ; 22}^{r}}+\sum_{l} \frac{1}{\mathbf{g}_{m m ; 22}^{r-1}-\mathbf{\Sigma}_{m m ; 22}^{r}} \boldsymbol{\Sigma}_{m l ; 21}^{r} \mathbf{G}_{l n ; 12}^{r} .
$$

With $\hat{\mathbf{G}}_{m n}^{r}$ and $\hat{\mathbf{G}}_{m n}^{<}$solved, from Eq. (17) the ac component and time-dependent current can be calculated without further complications.

In the rest of this section, we present numerical results for which some further simplifications are made. We reduce the device-normal region to a quantum dot with a spindegenerate single level, i.e., $H_{c e n}=\Sigma_{\sigma} \epsilon_{d} c_{\sigma}^{\dagger} c_{\sigma}$. For this case the boldface matrices reduce to a $C$ number. We also take $\Delta=\Delta_{L}=\Delta_{R}=1$ as the energy unit and only consider devices with symmetric barriers $\left(\Gamma_{L}=\Gamma_{R}\right)$. It should be mentioned that since we have assumed a spin-independent intradot level $\epsilon_{d}$ and hopping elements $t_{L(R)},\left\langle c_{\uparrow}^{\dagger}\left(t_{1}\right) c_{\uparrow}(t)\right\rangle$ should be equal to $\left\langle c_{\downarrow}^{\dagger}\left(t_{1}\right) c_{\downarrow}(t)\right\rangle$. Following this we have $G_{11}^{<}\left(t, t_{1}\right)$ $+G_{22}^{<}\left(t_{1}, t\right)=-\left[G_{11}^{r}\left(t, t_{1}\right)-G_{11}^{a}\left(t, t_{1}\right)\right] \quad$ and $\quad \hat{G}^{<}\left(t, t_{1}\right)$ $=-\left[\hat{G}^{<}\left(t_{1}, t\right)\right]^{\dagger} .{ }^{33}$ The Fourier forms are $G_{m n ; 11}^{<}(\epsilon)$ $+G_{-n,-m ; 22}^{<}(-\epsilon)=-\left[G_{m n ; 11}^{r}(\epsilon)-G_{n m ; 11}^{r *}(\epsilon)\right]$ and $\hat{G}_{n m}^{<}(\epsilon)$ 


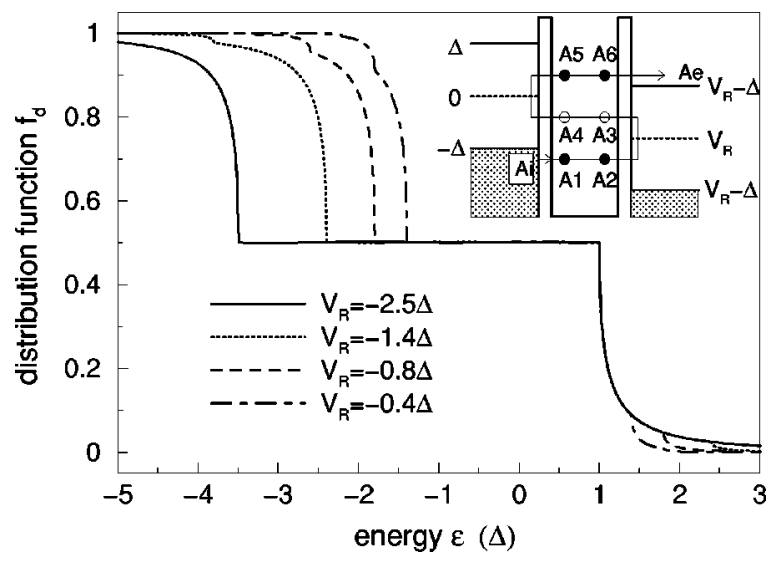

FIG. 2. Time-averaged intradot distribution of electrons versus energy $\epsilon$ at large $\Gamma, \Gamma_{L}=\Gamma_{R}=1000 \Delta$. Temperature $K_{B} \mathcal{T}=0.05 \Delta$, $\epsilon_{d}=0, \delta=0$ ( $\delta$ is the inelastic scattering rate in two superconducting leads), and $\phi_{L}=\phi_{R}=0$. Note the fact that the timeaveraged distribution, LDOS, and the ac components of the current are all independent with initial values of $\phi_{L}$ and $\phi_{R}$ at $\delta=0$. Inset: schematic diagram showing a multiple (two) Andreev reflection process.

$=-\left[\hat{G}_{m n}^{<}(\epsilon)\right]^{\dagger}$. These relationships provide very strong checks on our analytical derivations and numerical calculations which we present in the following sections.

\section{A. Intradot distribution of electrons}

In this section we present results of the intradot distribution of electrons for the $S$-normal- $S$ device. Owing to the finite bias voltage $V$, the current, intradot occupation number of electrons, LDOS, and the intradot distribution of electrons, are all functions of the time $t$. The time-average occupation number of electrons on the intradot state $\uparrow$ is (same for state $\downarrow$ )

$$
\left\langle n_{\uparrow}(t)\right\rangle_{t}=-i\left\langle G_{11}^{<}(t, t)\right\rangle_{t}=-i \int \frac{d \epsilon}{2 \pi} G_{00 ; 11}^{<}(\epsilon) .
$$

The integrand of Eq. (39), $(-i / 2 \pi) G_{00 ; 11}^{<}(\epsilon)$, is the timeaveraged occupation number of electrons with energy $\epsilon$. Here, subscript " 11 " are indexes of the $2 \times 2$ Nambu matrix element, and " 00 " are indexes of Fourier component. The average LDOS is given by $D(\epsilon)=-(1 / \pi) \operatorname{Im}\left[G_{00 ; 11}^{r}(\epsilon)\right.$ $\left.+G_{00 ; 22}^{r}(-\epsilon)\right]$. The average intradot distribution of electrons can be obtained from the average occupation number at energy $\epsilon$ and the average $D(\epsilon),{ }^{34}$

$$
f_{d}(\epsilon)=\frac{i G_{00 ; 11}^{<}(\epsilon)}{2 \operatorname{Im}\left[G_{00 ; 11}^{r}(\epsilon)\right]} .
$$

It is important to emphasize that the distribution of electrons can be experimentally measured. ${ }^{35,36}$ For example, recently Pierre et al. have measured ${ }^{36}$ this distribution for a $S$-normal$S$ device where the normal region is a diffusive mesoscopic metallic wire. They reported a multiple-step structure for the distribution of electrons in that device. ${ }^{36}$

Figure 2 shows the average intradot distribution of elec- trons at different bias voltage $V$ for our system with a very large coupling $\Gamma$. When $\Gamma$ is large, coupling between the superconducting leads and the normal region is strong, therefore the device behaves in a manner similar to a $S$-ballisticnormal-conductor- $S$ system. The property of the electron distribution in this situation is the following. When $\min \left(V_{L}\right.$ $\left.-\Delta, V_{R}-\Delta\right)<\epsilon<\max \left(V_{L}+\Delta, V_{R}+\Delta\right)$, the distribution is a constant, i.e., $f_{d}(\epsilon) \sim 1 / 2$ for symmetric couplings. When $\epsilon$ goes away from this region, the distribution quickly rises (or drops) to unity (or to zero) for $\epsilon<\min \left(V_{L}-\Delta, V_{R}-\Delta\right.$ ) [or for $\left.\epsilon>\max \left(V_{L}+\Delta, V_{R}+\Delta\right)\right]$.

To contrast with the experimental results of Pierre et al. ${ }^{36}$ and the theoretical results of Bezuglyi et al.,${ }^{37}$ here the distribution is a constant instead of the multiple-step structure between the gap, even though multiple Andreev reflections do occur in our system. This difference originates from the different property of the central device region, i.e., our normal region is ballistic while that in Pierre et al. experiment it is diffusive ${ }^{36}$ In order to explain it in more detail, the inset of Fig. 2 shows a particular multiple (two) Andreev reflection process. To start, an incident electron at $A_{i}$ below the gap of the left lead tunnels into the QD, it passes two Andreev reflections (through the points labeled as $A 1-A 6$ ) inside the QD and finally tunnels into the right lead (at $A_{e}$ ) which is higher than the gap of the right lead. Due to the ballistic nature of the QD, the distribution of electrons at point $A 1$ is the same as at $A 2$, the distribution of holes at $A 3$ is the same as at $A 4$, while distribution of electrons at $A 5$ is the same as at $A 6$. When $\Gamma$ is large, the probability of Andreev reflection inside the QD within the energy gap is very close to unity, ${ }^{38}$ and hence the hole distribution at $A 3$ is, to a very good extent, the same as the distribution of electrons at $A 2$. Similarly the hole distribution at $A 4$ is approximately the same as the electron distribution at $A 5$. We hence conclude that for the ballistic normal region, the distribution of particles (electrons and holes) along this path is the same everywhere, except at the abrupt change during the tunneling process at $A_{i}$ and $A_{e}$ from and to the two leads. Moreover, for symmetric barriers, the distribution of particles along the $A 1-A 6$ path must be $1 / 2$. This explains why we obtained a constant $1 / 2$ distribution at $\min \left(V_{L}-\Delta, V_{R}-\Delta\right)<\epsilon<\max \left(V_{L}\right.$ $\left.+\Delta, V_{R}+\Delta\right)$ as shown in Fig. 2. This also explains why we expect a different distribution when the normal region is diffusive: for a diffusive conductor the distribution at $A 1$ and $A 2$ must be different due to diffusive scattering between the two points, therefore the distribution of particles will continuously vary from one to zero along the path A1-A6.

Next, we investigate the distribution of electrons for $\Gamma \sim \Delta$, the results are shown in Fig. 3. For this case, a most prominent behavior of $f_{d}(\epsilon)$ is that it oscillates as a function of the $\epsilon$. The oscillations also become more rapid when bias voltage $V$ is reduced. An oscillatory $f_{d}(\epsilon)$ means its value is not necessarily smaller for larger $\epsilon$, hence a "population inversion" is possible. This population inversion originates from the nonmonotonic probability of Andreev reflections. For example, $f_{d}(\epsilon)$ has a dip at $\epsilon=V_{R}-\Delta$, due to the following reason. For an incident electron coming from the left lead with energy $V_{R}-\Delta$, this electron has a small but non- 


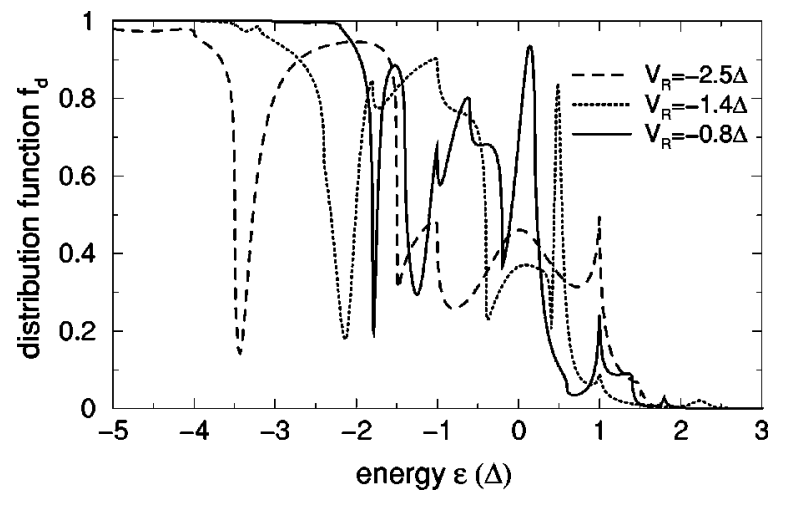

FIG. 3. Time-averaged intradot distribution of electrons versus energy $\epsilon$ at general QD parameters, $\Gamma_{L}=\Gamma_{R}=1.5 \Delta$. Other parameters are the same as those of Fig. 2.

zero probability to pass the left barrier. After tunneling through, it reaches the right barrier where an Andreev reflection occurs. Because this electron has energy $\epsilon=V_{R}-\Delta$, the Andreev reflection occurs with probability one. ${ }^{38}$ This means that the electron with energy $V_{R}-\Delta$ is difficult to tunnel into the QD, but it is easy to leave the QD after tunneling into it. Therefore, the distribution of electrons at this energy $\epsilon$ is very small. When $\epsilon$ deviates from $V_{R}-\Delta$, the probability of Andreev reflection decreases leading to a larger $f_{d}$, hence we expect a dip in $f_{d}$ to emerge at $\epsilon=V_{R}-\Delta$.

\section{B. Local density of states}

In this section, we investigate another important quantity, the average LDOS. We will mainly discusses Andreev resonance states at a finite bias $V \cdot{ }^{39,40}$ If bias $V>2 \Delta$, multiple Andreev reflections are very weak hence no Andreev resonance states can form in the QD. In this case the intradot level $\epsilon_{d}$ is only slightly shifted due to a nonzero real part of the self-energy $\Sigma^{r}$, the level half-width is still on the scale of $\Gamma_{L / R}$, and extra structures (a dip and a peak) emerge in the curves of $D(\epsilon)$ versus $\epsilon$ at the superconducting gap (not shown in here).

Much more interesting is the case of $V<\Delta$, shown in Fig. 4 at different bias $V$. A series of very narrow peaks emerge in $D(\epsilon)$, clearly indicating the formation of Andreev resonance states inside the QD. Note that they are not rigorous bound states but are quasibound states with a finite lifetime, and after many Andreev reflections the particle can leave the QD. The half-width of Andreev resonance states is much narrower than $\Gamma$. With a decreasing bias $V$, they become even narrower with a higher intensity. The average distance between two successive Andreev resonance states is about $e V$. When $n e V$ and $(n+1) e V(n=0, \pm 1, \pm 2, \ldots)$ are within the gap, there exists an Andreev resonance state between $\epsilon$ $=n e V$ and $(n+1) e V$. Moreover, these Andreev resonance states are symmetrically distributed at the two sides of $V_{L}$ and $V_{R}$. This means the following: when an incident electron from below the gap aligns perfectly with an Andreev resonance state of the QD, even after many Andreev reflections it will always stay on the Andreev resonance state until it leaves the QD [see inset of Fig. 4(a)]. Along this path, the

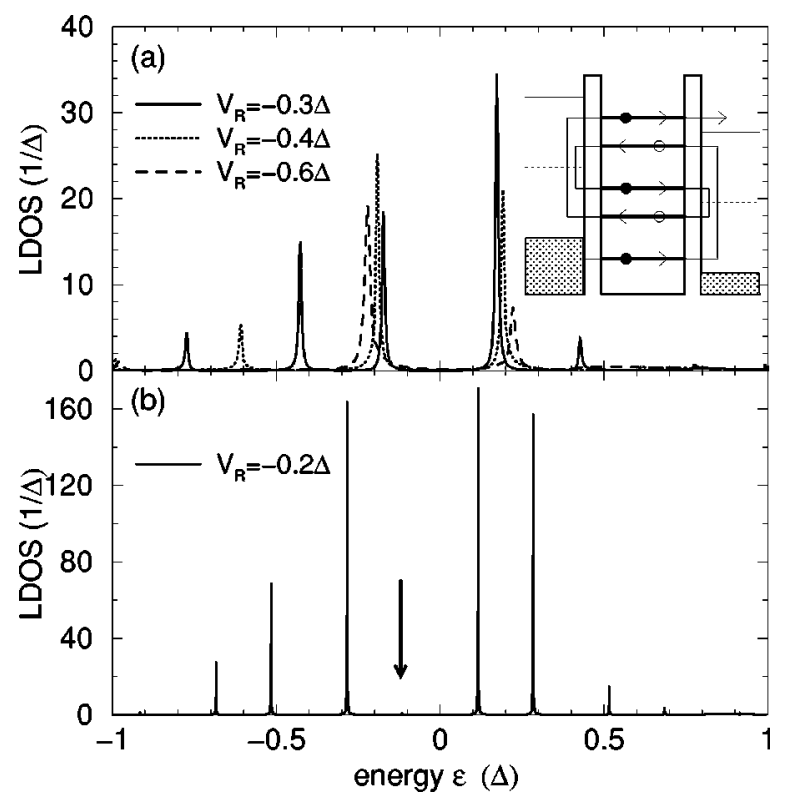

FIG. 4. Time-averaged LDOS versus $\epsilon$ at different bias $V$. $K_{B} \mathcal{T}=0.1 \Delta, \Gamma_{L}=\Gamma_{R}=0.8 \Delta, \epsilon_{d}=0$, and $\delta=0$. The downward arrow in (b) points to an Andreev resonance state with a very small LDOS. Inset in (a): schematic diagram showing a multiple Andreev reflection that passes through the Andreev resonance states indicated by the thick solid lines in the QD.

particle goes through all Andreev resonance states, and a resonance multiple Andreev reflection occurs. Occasionally, a quenching of Andreev resonance state is observed to occur. In this case, a specific Andreev resonance state may have very low LDOS at a specific bias $V$, an example is indicated by the arrow in Fig. 4(b).

The results of Fig. 4 is obtained by fixing the intradot level $\epsilon_{d}$ to zero (i.e., at the center of the gap). Next, we investigate how Andreev resonance states are affected when $\epsilon_{d} \neq 0$, the results are shown in Fig. 5. With $\epsilon_{d} \neq 0$, the Andreev resonance states are shifted in their positions, but their physical characteristics are the same as those of $\epsilon_{d}=0$. The amount of shift is not $\epsilon_{d}$ but much smaller and two successive Andreev resonance states are shifted in opposite directions. If an Andreev resonance state is in the energy range

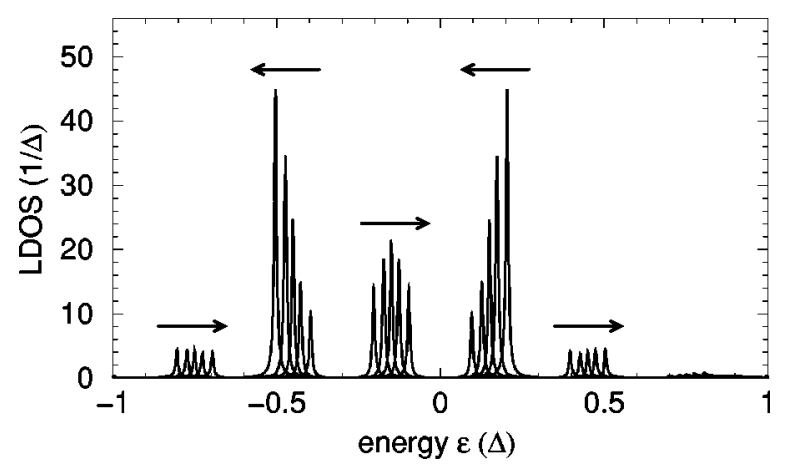

FIG. 5. Time-averaged LDOS versus $\epsilon$ at different level positions $\epsilon_{d} . V_{R}=-0.3 \Delta$ and other parameters are the same as those of Fig. 4. Different curves correspond to $\epsilon_{d}=0.15 \Delta, 0 \Delta,-0.15 \Delta$, $-0.30 \Delta$, and $-0.45 \Delta$, along the arrow direction. 


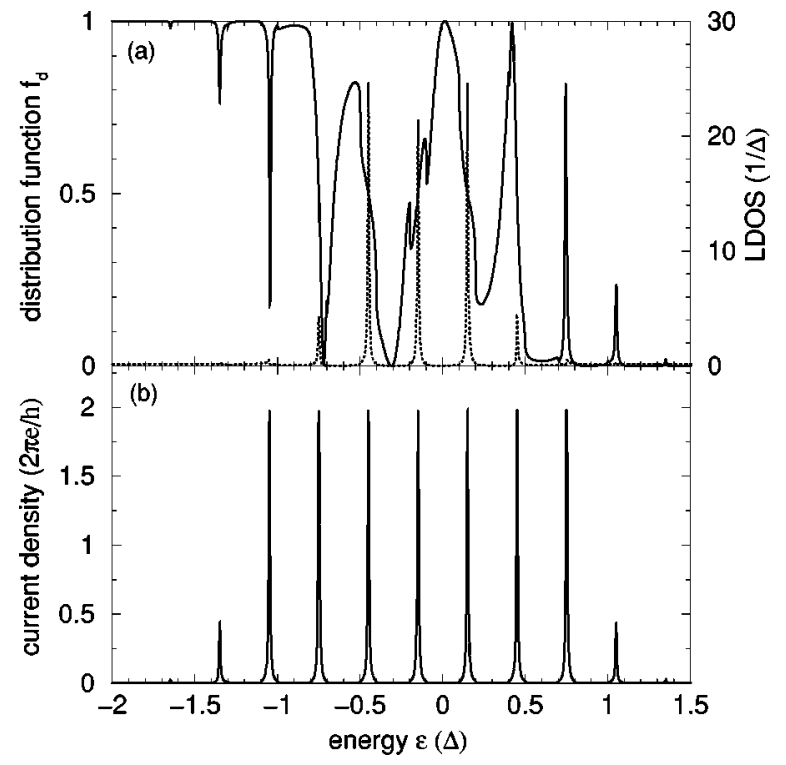

FIG. 6. (a) Time-averaged LDOS (dotted) and the time-averaged distribution of electrons (solid) versus $\epsilon$; (b) the time-averaged current density. $\epsilon_{d}=-0.15$ and other parameters are the same as those in Fig. 5.

from $\epsilon=n e V$ to $(n+1) e V$, it stays in this range at any value of $\epsilon_{d}$. Their heights vary with $\epsilon_{d}$, when $\epsilon_{d}$ is in the range of $n e V$ to $(n+1) e V$, the peak in this range reaches a maximum value.

An important property of the Andreev resonance states is their ability to carry current. From Eqs. (16) and (17), the time-averaged current density $j_{0}(\epsilon)$ is obtained to be

$$
\begin{aligned}
j_{0}(\epsilon)= & -\frac{e}{\pi} \operatorname{Im} \operatorname{Tr}\left\{\left[f_{L}(\epsilon) \tilde{\rho}_{L}(\epsilon) \hat{G}_{00}^{r}(\epsilon)\right.\right. \\
& \left.\left.+\frac{1}{2} \beta_{L}^{*}(\epsilon) \hat{G}_{00}^{<}(\epsilon)\right] \Gamma_{L} \hat{\tilde{\Sigma}}_{L} \hat{\sigma}_{z}\right\} .
\end{aligned}
$$

The current density is related to time-averaged current as $I_{0}=\int d \epsilon j_{0}(\epsilon)$. In Fig. 6, we show intradot distribution of electrons $f_{d}$ [solid curve in Fig. 6(a)], LDOS [dotted curve in Fig. 6(a)], and the time-averaged current density [Fig. 6(b)] $j_{0}(\epsilon)$. Several observations are in order.

(i) Although $f_{d}(\epsilon)$ is oscillating between 0 and 1 in a complicated manner, its value at each Andreev resonance state (the peak positions of the dotted curve) is always $1 / 2$. This is because resonant multiple Andreev reflections occur along the path of Andreev resonance states [as shown in the inset of Fig. 4(a)].

(ii) The current density $j_{0}(\epsilon)$ is dominated by a series of peaks located precisely at the energies of Andreev resonance states. This is a clear indication that current is carried by Andreev resonance states. When $\min \left(V_{L}-\Delta, V_{R}-\Delta\right)<\epsilon$ $<\max \left(V_{L}+\Delta, V_{R}+\Delta\right)$, the peaks of $j_{0}(\epsilon)$ all have the same height: this means each Andreev resonance state carries exactly the same amount of current in the same flow direction. The reason for this peculiar behavior is simple. Along the path of Andreev resonance states [inset of Fig. 4(a)], all the electrons move in one direction while all the holes move in

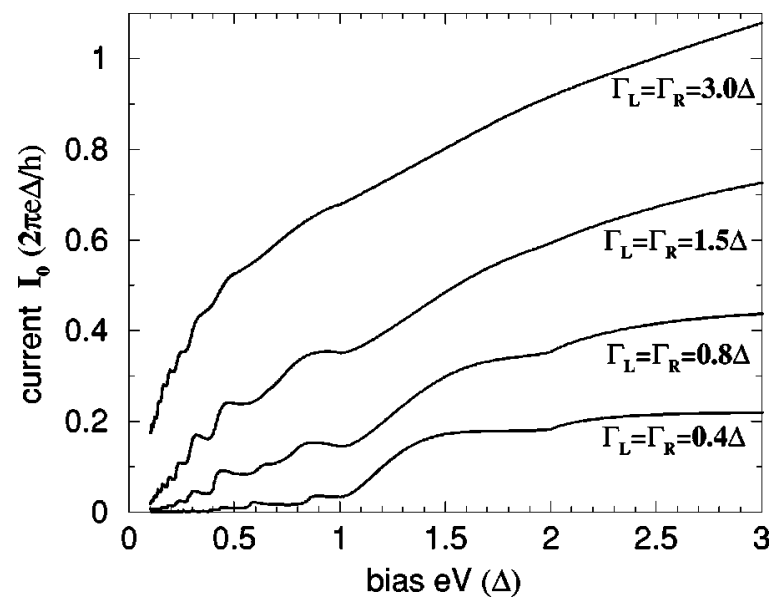

FIG. 7. Time-averaged current $I_{0}$ versus bias $V$ at different $\Gamma$. Other parameters: $K_{B} \mathcal{T}=0.1 \Delta, \epsilon_{d}=0, \delta=0.005 \Delta, \phi_{L}=\phi_{R}=0$.

opposite direction, and along any one path the particle current must be the same everywhere. Therefore, the Andreev resonance states carry the same amount of current in the same direction.

\section{The current}

The time-averaged current $I_{0}$ of $S$-normal- $S$ systems has been extensively investigated both theoretically and experimentally. A main characteristic of the $I-V$ curve $I_{0}(V)$ is its subharmonic gap structure at $V=2 \Delta / n,{ }^{3-8,41}$ our results are shown in Fig. 7. The $I-V$ curves also exhibit subharmonic gap structure with a concomitant appearance of negative differential conductance. These results are in agreement with those reported recently by Yeyati et al. ${ }^{6}$ and Johansson et $a l .{ }^{10}$ In the following, we focus on the ac component of the current.

From Eqs. (13) and (16), we decompose the timedependent current into its dissipative contribution $I_{n}^{c}$, and nondissipative contribution $I_{n}^{s},{ }^{4}$

$$
I_{L}(t)=I_{0}+\sum_{n} I_{L n}^{c} \cos n \omega t+\sum_{n} I_{L n}^{s} \sin n \omega t
$$

where $I_{L n}^{c} \equiv \operatorname{Im}\left(\mathcal{I}_{L n}+\mathcal{I}_{L-n}\right)$ and $I_{L n}^{S} \equiv \operatorname{Re}\left(\mathcal{I}_{L n}-\mathcal{I}_{L-n}\right)$. Figure 8 and 9 show the first and second ac components of $I_{L n}^{c}$ and $I_{L n}^{S}$ as a function of the bias $V$, and they are marked by a strong oscillatory behavior. The period of oscillations is roughly given by $V^{2} / \Delta$, which is dependent on bias $V$. Generally, for $2 \Delta / m<e V<2 \Delta /(m+1)(m=1,2, \ldots)$, we found that the ac components oscillate from a maximum to a minimum or vice versa. When $V>2 \Delta / n$, the components $I_{L n}^{c}$ and $I_{L n}^{s}$ quickly decay to zero. When $e V \sim \Delta / n$, the amplitudes of the oscillations reach maximum. At $e V \rightarrow 0, I_{L n}^{c}$ decays to zero while $I_{L n}^{S}$ keeps a finite value. These behaviors are different from those devices whose normal region has no electronic structure. For instance, the result of $S$-QPC- $S$ system shows no oscillation. ${ }^{4}$ 


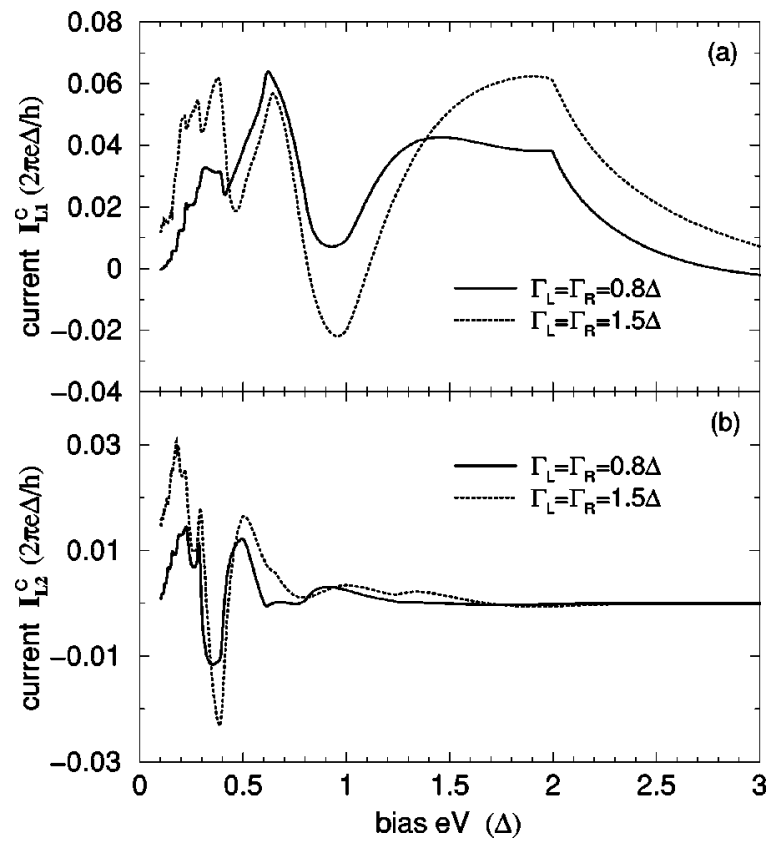

FIG. 8. Dissipative ac components $I_{L 1}^{c}$ and $I_{L 2}^{c}$ versus bias $V$ at different $\Gamma$. Other parameters are the same as those of Fig. 7.

The time-dependent current $I_{L}(t)$ is shown in Fig. 10 . $I_{L}(t)$ is a well-known oscillatory function of the time $t$ with a frequency $\omega=2 \mathrm{eV}$. When bias $V$ is large, $e V>\Delta$, the high-order Fourier components have negligible contribution and $I_{L}(t)$ can be approximated by $I_{L}(t) \approx I_{0}+I_{L 1} \sin (\omega t$ $+\phi)$. On the other hand, when $V$ is small, high-order components contribute substantially and $I_{L}(t)$ deviates from a simple sinelike curve.

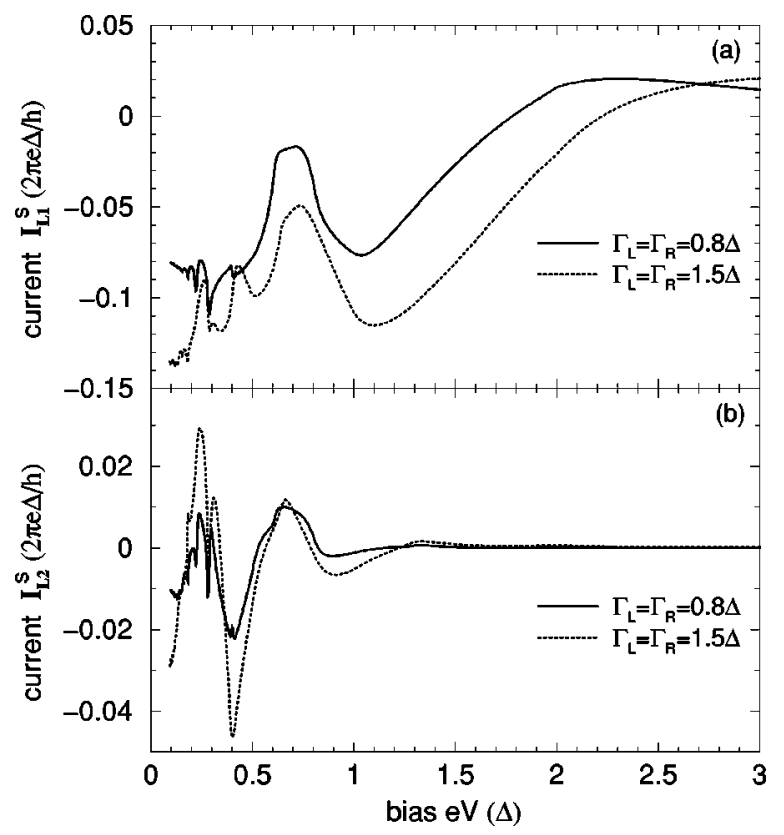

FIG. 9. Nondissipative ac components $I_{L 1}^{s}$ and $I_{L 2}^{s}$ versus bias $V$ at different $\Gamma$. Other parameters are the same as those of Fig. 7.

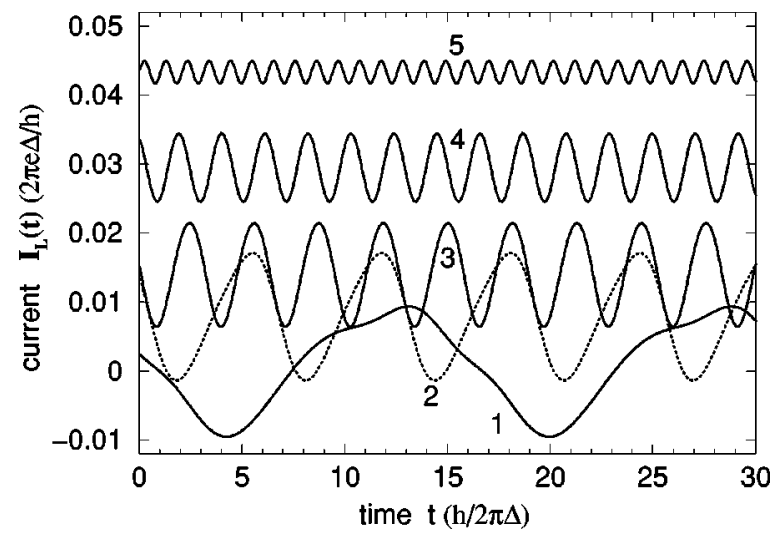

FIG. 10. Time-dependent current $I_{L}(t)$ versus time $t$ at different bias $V . \Gamma_{L}=\Gamma_{R}=0.8 \Delta$ and other parameters are the same as those of Fig. 7. The curves labelled $1-5$ correspond to $V=-V_{R}=0.2 \Delta$, $0.5 \Delta, 1.0 \Delta, 1.5 \Delta$, and $3.0 \Delta$, respectively.

\section{CONCLUSIONS}

In this work, we have derived a general formula for ac components of the time-dependent current of arbitrary ballistic $S$-normal-S systems where the normal region has its own electronic structure. The formula [Eq. (17)] is valid even when there is a strong interaction in the normal region of the hybrid device. We then applied this result to study ac Josephson current for a system with the normal region being a noninteracting single-level quantum dot. The average intradot distribution of electrons, the average intradot density of states, and ac components of the time-dependent current are investigated in detail. The distribution exhibits an interesting population inversion, a result that is qualitatively different from that of the diffusive normal region. A series of Andreev resonance states are formed at bias $V<\Delta$ in our system. The peak heights of LDOS for these Andreev resonance states are not the same, but each state carries the same amount of current. The distribution of electrons at the Andreev resonance states are all the same, e.g., equals $1 / 2$ for symmetric tunnel barriers. In general, the ac components of the time-dependent current has an oscillatory behavior against the bias. Depending on the value of bias, the high-order ac components may or may not contribute to the total time-dependent current, leading to a non-sinelike or a sinelike dependence on time for the total current.

Finally, we comment on the $e V \rightarrow 0$ limit for the $S$-QD-S system of this work. While our general current formula, Eq. (17), is valid for any bias, how to correctly include important physical factors in an actual computation of the various quantities of Eq. (17), needs to be discussed. When bias is very small, $e V \ll \Delta$, an incident electron from below the gap of the left superconducting lead undergoes many Andreev reflections in the QD so as to go above the gap of the right superconducting lead before exiting the QD. Therefore, the dwell time $\tau_{p}$ of the particle in the QD becomes long. At the limit $e V \rightarrow 0, \tau_{p}$ tends to large values. When $\tau_{p}$ is larger than the mean inelastic scattering time, the intradot relaxation effect should be considered in calculating the Green's functions involved in Eq. (17). When there is no electronic structure in the normal region of the device, for instance in a 
$S$-QPC- $S$ system, ${ }^{4,5}$ the $e V \rightarrow 0$ limit has a variety of different regimes depending on an inelastic scattering-rate parameter $\delta$ and a transmission probability of the QPC. ${ }^{4,5}$ For our $S$-QD- $S$ system, while relaxation in the leads can similarly be included by introducing the same parameter $\delta$ into the Green's function of the leads, ${ }^{4}$ this simple phenomenological approach cannot be applied in the normal QD region. This is because distribution of leads is determined by their chemical potential; however, the distribution in the QD must be calculated self-consistently for our system. Indeed, if one introduces a finite $\delta$ in the QD Green's function, current conservation will be violated. A proper treatment of this problem is, perhaps, to explicitly introduce an electron-phonon interac- tion term in the Hamiltonian. This is a very complicated problem to solve and we hope to be able to report such an analysis in the future.

\section{ACKNOWLEDGMENTS}

We gratefully acknowledge financial support from NSERC of Canada, FCAR of Québec (Q.S. and H.G.), and for a RGC grant from the SAR Government of Hong Kong under Grant No. HKU 7215/99P (J.W.). Q.S. thanks Y. Liu and X.B. Zhu for their help on the numerical calculations.
${ }^{1}$ B.J. van Wees and H. Takayanagi, in Mesoscopic Electron Transport, edited by L.L. Sohn, L.P. Kouwenhoven, and G. Schön (Kluwer, Dordrecht, 1997).

${ }^{2}$ F.W.J. Hekking, G. Schon, and D.V. Averin, Physica B 203, 201 (1994).

${ }^{3}$ E.N. Bratus, V.S. Shumeiko, and G. Wendin, Phys. Rev. Lett. 74, 2110 (1995).

${ }^{4}$ J.C. Cuevas, A. Martin-Rodero, and A.L. Yeyati, Phys. Rev. B 54, 7366 (1996).

${ }^{5}$ E.N. Bratus, V.S. Shumeiko, E.V. Bezuglyi, and G. Wendin, Phys. Rev. B 55, 12666 (1997).

${ }^{6}$ A.L. Yeyati, J.C. Cuevas, A. Lopez-Davalos, and A. MartinRodero, Phys. Rev. B 55, R6137 (1997).

${ }^{7}$ T.I. Ivanov, Phys. Rev. B 59, 169 (1999).

${ }^{8}$ K. Kang, Phys. Rev. B 57, 11891 (1998).

${ }^{9}$ S. Ishizaka, J. Sone, and T. Ando, Phys. Rev. B 52, 8358 (1995).

${ }^{10}$ G. Johansson, E.N. Bratus, V.S. Shumeiko, and G. Wendin, Phys. Rev. B 60, 1382 (1999).

${ }^{11}$ A. Golub and B. Horovitz, Phys. Rev. B 50, 15882 (1994).

${ }^{12}$ A.Yu. Kasumov, R. Deblock, M. Kociak, B. Reulet, H. Bouchiat, I.I. Khodos, Yu.B. Gorbatov, V.T. Volkov, C. Journet, and M. Burghard, Science 284, 1508 (1999).

${ }^{13}$ A.F. Morpurgo, J. Kong, C.M. Marcus, and H. Dai, Science 286, 263 (1999).

${ }^{14}$ Y. Wei, J. Wang, H. Guo, H. Mehrez, and C. Roland, Phys. Rev. B 63, 195412 (2001).

${ }^{15}$ P.F. Bagwell, Phys. Rev. B 46, 12573 (1992).

${ }^{16}$ J.J.A. Baselmans, A.F. Morpurgo, B.J. van Wees, and T.M. Klapwijk, Nature (London) 397, 43 (1999).

${ }^{17}$ E. Scheer, N. Agrait, J.C. Cuevas, A.L. Yeyati, B. Ludoph, A. Martin-Rodero, G.R. Bollinger, and J.M. Van, Nature (London) 394, 154 (1998).

${ }^{18}$ E. Scheer, W. Belzig, Y. Naveh, M.H. Devoret, D. Esteve, and C. Urbina, Phys. Rev. Lett. 86, 284 (2001).

${ }^{19}$ G.B. Arnold, J. Low Temp. Phys. 68, 1 (1987).

${ }^{20}$ D. Averin and A. Bardas, Phys. Rev. Lett. 75, 1831 (1995).

${ }^{21}$ M. Hurd, S. Datta, and P.F. Bagwell, Phys. Rev. B 56, 11232 (1997)

${ }^{22}$ M. Hurd, S. Datta, and P.F. Bagwell, Phys. Rev. B 54, 6557 (1996)
${ }^{23}$ T. Lofwander, G. Johansson, M. Hurd, and G. Wendin, Phys. Rev. B 57, R3225 (1998).

${ }^{24}$ M. Hurd, T. Lofwander, G. Johansson, and Wendin, Phys. Rev. B 59, 4412 (1999).

${ }^{25}$ Q.-f. Sun, J. Wang, and T.-h. Lin, Phys. Rev. B 62, 648 (2000).

${ }^{26}$ Q.-f. Sun, B.-g. Wang, J. Wang, and T.-h. Lin, Phys. Rev. B 61, 4754 (2000).

${ }^{27}$ N. S. Wingreen, Antti-Pekka Jauho, and Y. Meir, Phys. Rev. B 48, 8487 (1993).

${ }^{28}$ Antti-Pekka Jauho, N. S. Wingreen, and Y. Meir, Phys. Rev. B 50, 5528 (1994).

${ }^{29}$ If the inelastic processes inside the superconducting lead are considered, $\epsilon$ will have a small positive imaginary part $\delta$, then $\beta_{L}(\epsilon+i \delta)=(\epsilon+i \delta) /\left[i \sqrt{\Delta_{L}^{2}-(\epsilon+i \delta)^{2}}\right]$ for $\Delta_{L}>|\epsilon|$, and $\beta_{L}(\epsilon$ $+i \delta)=\left[(\epsilon+i \delta) \operatorname{Sgn}(\epsilon) /\left[\sqrt{(\epsilon+i \delta)^{2}-\Delta_{L}^{2}}\right]\right.$ for $\Delta_{L}<|\epsilon|$.

${ }^{30}$ Q.-f. Sun, J. Wang, and T.-h. Lin, Phys. Rev. B 59, 13126 (1999).

${ }^{31}$ J. Wang, Y. Wei, H. Guo, Q.-f. Sun, and T.-h. Lin, Phys. Rev. B 64, 104508 (2001).

${ }^{32}$ In this expression, the condition of $V_{L}=0$ has been used. If $V_{L}$ $\neq 0, G_{12}\left(t, t_{1}\right)$ may be not equal to $G_{12}\left(t+T, t_{1}+T\right)$.

${ }^{33}$ Even if the intradot level $\epsilon_{d}$ and the hopping elements $t_{L(R)}$ are dependent on spin index $\sigma$, the equation $\hat{G}^{<}\left(t, t_{1}\right)=$ $-\left[\hat{G}^{<}\left(t_{1}, t\right)\right]^{\dagger}$ is still valid.

${ }^{34}$ Strictly speaking, the time-averaged distribution of electrons should be calculated as follows: first one calculates the ratio of time-dependent occupation number and the LDOS; second one performs the time average of this ratio. However, due to the fact that high-order components of the distribution are generally small, (except at small bias for those devices with large $\Gamma$ ), the distribution can be well approximated by the ratio of average quantities.

${ }^{35}$ H. Pothier, S. Gueron, N.O. Birge, D. Esteve, and M.H. Devoret, Phys. Rev. Lett. 79, 3490 (1997).

${ }^{36}$ F. Pierre, A. Anthore, H. Pothier, C. Urbina, and D. Esteve, Phys. Rev. Lett. 86, 1078 (2001).

${ }^{37}$ E.V. Bezuglyi, E.N. Bratus, V.S. Shumeiko, G. Wendin, and H. Takayanagi, Phys. Rev. B 62, 14439 (2000).

${ }^{38}$ G.E. Blonder, M. Tinkham, and T.M. Klapwijk, Phys. Rev. B 25, 4515 (1982); M. Octavio, M. Tinkham, G.E. Blonder, and T.M. Klapwijk, ibid. 27, 6739 (1983).

${ }^{39}$ It is worth mentioning that at a finite bias $V$, Hamiltonian $H$ is 
dependent with time $t$, and the system is in a nonstationary state. Therefore, the energy $\epsilon$ is not a good quantum number. However, due to $H(t)=H(t+T)$ being a periodic function of $t$, the system's state can have the form $\Phi(t)=e^{-i E t} \varphi(t)$, where $E(0 \leqslant E<\omega)$ is the quasienergy and $\varphi(t+T)=\varphi(t)$ is the Floquet state. $\varphi(t)$ can be expanded, $\varphi(t)=\Sigma_{n} A_{n} e^{-i n \omega t}$ and $\Phi(t)$ $=\Sigma_{n} A_{n} e^{-i(E+n \omega) t}$, so that a quasienergy state seems to consist of a series of eigenstates with energy $E+n \omega$ with weight $A_{n}$. The LDOS discussed in the text is the density of these eigen- states, i.e., the density of the quasienergy $E(0 \leqslant E<\omega)$ state is $\sum_{n} D(E+n \omega)$.

${ }^{40}$ P. Samuelsson, J. Lantz, V.S. Shumeiko, and G. Wendin, Phys. Rev. B 62, 1319 (2000).

${ }^{41}$ A.W. Kleinsasser, R.E. Miller, W.H. Mallison, and G.B. Arnold, Phys. Rev. Lett. 72, 1738 (1994); N. van der Post, E.T. Peters, I.K. Yanson, and J.M. van Ruitenbeek, ibid. 73, 2611 (1994). 